\title{
Rethinking the Design of Robotic Pets for Older Adults
}

\author{
Amanda Lazar ${ }^{1}$, Hilaire J Thompson ${ }^{2}$, Anne Marie Piper ${ }^{1}$, George Demiris ${ }^{2}$ \\ ${ }^{1}$ Northwestern University, Evanston, IL; ${ }^{2}$ University of Washington, Seattle, WA \\ \{lazar,ampiper\}@northwestern.edu, \{hilairet,gdemiris\}@uw.edu
}

\begin{abstract}
Robots are seen as a potential solution to the perceived needs of the aging population. Thus far, research has primarily focused on robotics for the functional and emotional support of older adults. Robotic pets have been developed primarily for the older adult who is perceived as lonely and isolated, and fears have consequently arisen that robots will replace human caregivers and deceive older adults into developing relationships with them. Missing is the perspective of older adults on the ethics of and potential uses for robotic companion pets. In this study, we conducted focus groups with 41 older adults. We discuss concepts raised by focus group participants such as giving into the fiction of the robotic pet, the social role of the robot, and the role of reciprocity in building a relationship with a robotic pet. We present resulting considerations for new directions for robotic pet design for older adults.
\end{abstract}

\section{Author Keywords}

Aging; older adults; robots

\section{ACM Classification Keywords}

H.5.m. Information interfaces and presentation (e.g., HCI): Miscellaneous.

\section{INTRODUCTION}

As the population ages worldwide, healthcare costs will rise and a shortage of caregivers is foreseen. In response to these concerns, robots are frequently cited as the solution for some of the unmet needs of older adults [47]. Robots designed for older adults often fit into two categories: provision of functional support, such as delivering medications (e.g., Pearl the Nursebot [36]), or the alleviation of loneliness by embodying a snuggly, cuddly animal (e.g., Paro, a soft robotic seal designed for older adults).

In Sherry Turkle's popular TED Talk, she tells a story of seeing an older woman in a nursing home talk to Paro after her child had died. She said that as the woman was receiving

Permission to make digital or hard copies of all or part of this work for personal or classroom use is granted without fee provided that copies are not made or distributed for profit or commercial advantage and that copies bear this notice and the full citation on the first page. Copyrights for components of this work owned by others than the author(s) must be honored. Abstracting with credit is permitted. To copy otherwise, or republish, to post on servers or to redistribute to lists, requires prior specific permission and/or a fee.

Request permissions from Permissions@acm.org.

DIS 2016, June 04 - 08, 2016, Brisbane, QLD, Australia

Copyright is held by the owner/author(s). Publication rights licensed to ACM.

ACM 978-1-4503-4031-1/16/06..\$15.00

DOI: http://dx.doi.org/10.1145/2901790.2901811 comfort from Paro, Turkle thought that "... that woman was trying to make sense of her life with a machine that had no experience of the arc of a human life. That robot put on a great show. And we're vulnerable. People experience pretend empathy as though it was the real thing" [51]. Turkle describes this experience as one of the most "wrenching, complicated moments" in her career [51]. While Turkle and others (e.g. [15, 47]) raise concerns about the ethics of introducing robots into the lives of older adults, little research has examined older adults' views on how various types of robots could or should play a role in their lives.

Robots are often viewed as a "technological fix" for aging [17] and other populations deemed vulnerable [41], yet it is critical to understand older adults' perceptions and attitudes towards robots before designing with this population in mind. Currently, older adults are often "considered but not consulted" in the design of robots [20], and more work is needed to understand older adults' views on whether and how they envision interacting with robots in their daily lives.

To address this gap in the literature, we examine older adults' perspectives on robotic pets through eight focus groups with 41 participants. Drawing on their own experience with real pets, older adults in our study explored and discussed the idea of owning a robotic pet. Through this, our research asks: How do older adults perceive robotic pets? Which qualities of real pets should be inherited by robotic pets? How might robotic pets fit into an older adult's life in ways that complement or extend that of a real pet? Our analysis revealed three primary tensions in the design of and current narrative around robotic pets: (1) deriving comfort and companionship from a robotic pet requires giving into the fiction of it, which is an older adult's choice; (2) rather than alleviating an individual's loneliness and social isolation, robotic pets may provide social entertainment and facilitate opportunities for social interaction with others; and (3) the functional support of robotic pets is appealing, but older adults also desire the reciprocal nature of caring for a pet and the relationship this engenders.

This paper contributes to the literature on designing for older adults in ways that understand the full experience of aging rather than treating older adulthood as a time of decline and social isolation [53]. Additionally, the paper furthers the ongoing discussion of the ethics of introducing robots into the lives of older adults and offers insights and design directions for the robotics community.

\section{RELATED WORK}

Below we cover developments in robotics for older adults. Much of this work has focused on robots for functional 
support or companionship, and has involved older adults only peripherally.

\section{Socially Assistive Robots}

Social robots are robots that people anthromorphize to interact with and understand [7]. Assistive social robots for older adults can be categorized into service-type robots and companion robots [9]. Relevant to the present paper, robotic pets have the capacity to be both service and companion oriented (e.g. Matilda, a robot that plays Bingo as well as reminds people about their activities for the day [27]).

Service-type robots aid older adults in living independently through supporting activities such as eating and dressing mobility, and monitoring. For service-type robots, the social functions exist for users to interact with the robot, rather than for users to derive benefit from the social interaction. A significant amount of research on robots for older adults has focused on service robots. One specific theme of this research pertains to older adults' reactions to them. For example, researchers have assessed reactions to the appearance the PR2 robot after demonstrating its ability to perform three functional assistance tasks: delivering medication, clearing clutter, and operating a light switch [38]. In another study, robots provided medication to independently living older adults who were then interviewed about their experience, with the goal of helping older adults with medication adherence [37]. Research on service robots has also explored the ways these robots should serve the functional needs of older adults. Robotic products must fit the ecology of older adults' lives, support flexible use, and adapt to changes in functioning, while preserving older adults' values such as dignity and independence [19]. Our analysis contributes to this literature by understanding how robotic pets in particular could fit into older adults' lives.

Companion robots, on the other hand, interact with users to enhance their health and psychological wellbeing through companionship. Much of the research on companion robots has focused on the evaluation and usability testing of these robots, particularly in acute health care settings or for people with cognitive impairments [30,49]. Older adults in a care center enjoyed social interactions such as daily greetings from a robot operated by a researcher. They spontaneously told the robot about personal matters such as health and family issues and treated the robot as a child [43]. "My Real Babies," iRobot's robot in the form of a baby, served as a topic of conversation and were perceived by nursing home residents as a "grown-up" activity as opposed to non-robotic dolls [52]. Missing is a detailed analysis of which features makes a robot appear suitable for older adults, which is a topic we explore in this paper.

\section{Robotic Pets for Older Adults}

A substantial subsection of companion robots has been designed to look like animals, which we refer to as robotic pets. A possible reason for this is that pets cannot be present in certain settings such as hospitals [31] and are not compatible with certain lifestyles [2], yet they provide meaningful social interactions [31]. The majority of robotic pets designed for older adults have been developed for companionship (RIBA, a bear robot that lifts older adults [33] is a notable exception). Several studies have introduced Paro, a robotic seal, to nursing homes and have focused on the introduction and acceptance of this companion robot. People with cognitive impairments appear to be more willing to interact with Paro as time goes on [12]. Additionally, the role of scaffolding from staff and visitors [12] and individual histories and relationships [11] affects how older adults perceive and interact with Paro in group settings. One concept that has been proposed is introducing companion robots to nursing homes as shared objects with shared responsibility to care for them, as residents might be overwhelmed in caring for these robots [25].

Studies have also focused on positive benefits of using companion robots with older adults. Introducing Paro to a care home in Japan increased resident interactions and improved reactions to stress [54]. Researchers have explored how both live and robotic dogs (Sony's AIBO) relieved loneliness in long-term care residents and fostered attachment [2] and received similar amounts of attention from residents with dementia [28]. Robotic and stuffed animals have also been compared: people with dementia experience increased interest and pleasure using both robotic and stuffed cats [29]. However, explorations of robotic pet companions for older adults have largely focused on the reaction to a single robotic pet rather than understanding preferences for robotic pets that have not yet been developed. In contrast, our analysis examines variations across six example robotic pets in order to elicit preferences.

\section{Older Adults' Involvement in Robotics Research}

Although a growing body of literature focuses on the design and use of robots with older adults, few studies directly involve older adults. When they do it is often at the end stage of the design process (usability and evaluation), or through proxies such as caregivers (e.g. [23]), meaning that the older adult do not get to play a major role in the design [20]. An exception is Šabanović et al.'s work, which uses a participatory design approach to engage older adults with depression and chronic illness in the design process [42].

Most research that involves older adults in earlier stages of research focuses on older adults' perceptions and wishes, particularly in the area of functional assistance provided by robots. Researchers have explored independently living older adults' opinions of using a robot in the home and preferences for assistance from robots vs. humans for household maintenance [4]. Some research has found that older adults wish robots to provide social interaction in addition to functional assistance such as home upkeep [3, $10]$, though other research has found that older adults are not particularly interested in companionship from robots [8].

Even when older adults are involved throughout the development of a robot, their views may not be incorporated into the final design. Compagna and Kohlbacher take a 
critical perspective of the project that they were involved with as social scientists. They describe how, despite framing the study as involving residents in participatory design, designers ignored the preferences of the older adults throughout the design process, perceiving them as an "irrelevant user group" [14].

Few studies have solicited older adults' opinions of companion robots or robotic pets. McGlynn et al. interviewed and administered surveys to healthy older adults before and after they interacted with Paro to understand whether they saw the robot as useful to themselves and others [30]. In another study, six participants participated in focus groups before and after they were given companion robots, predominantly robotic pets, to use at home [24]. Participants were largely unsatisfied with the robotic pets and saw them as being more appropriate for other older adults "in need of intellectual stimulation, companionship, and empathy" and with limited independence/mobility. Although older adults in our study also saw robotic pets as for the "socially isolated older adult," they were able to move beyond this view to articulate how robotic pets could be appealing to themselves.

The perception of robotic pets as for the "socially isolated older adult" - held by the research community and older people themselves - furthers negative stereotypes of aging and neglects the diversity of experiences in older adulthood that robotic pets could support. Researchers have begun to reflect on the stereotypical views of older adults as lonely, passive, and in need of functional assistance [17, 20,34], which drive the design of robots as 'technological fixes' for older adults [20, 34, 41]. These stereotypical views, values, and social norms affect the technological capabilities of the robots that are created [41]. One case study which involved the observation of a set of developers illustrates this point [34]: the developers expected older adults to need and benefit from robots, especially for physical and emotional needs. Older adults who were involved in the evaluation of the robot perceived the intended user of this robot as a lonely and physically and mentally frail older adult and distanced themselves from that persona. The abundance of stereotypes and negative views of older adults is not exclusive to robotics. In HCI, aging is often framed as a problem technology can solve, and older people are often positioned as lonely and disengaged [53]. Additionally, research has largely devoted itself to compensate for perceived deficits rather than appeal to what older people actually want [40]. An understanding of what older adults want from robots, and, in particular, robotic companion pets, is missing from the literature and is the core contribution of this paper.

\section{METHODS}

\section{Focus Groups}

We conducted eight focus groups at four independent living communities and one community center. Forty-one older adults participated (age range 61-92; mean age 77; standard deviation $8.8 ; 35$ females). We intentionally chose independent living communities that spanned different rent rates and included subsidized housing to obtain participants at varying income levels. Thirty-five participants had owned pets at some point and four had not (two did not answer).

The goal of the focus groups was to explore older adults' understanding of what it means to have a robotic pet, their perceptions of robotic pets, and the ways they expressed interest or disinterest in interacting with pets. Participants were told that robotic pets are "interactive robots of different sizes that may look and act like existing pets, or appear like animals that don't exist and have capabilities that existing pets don't have," and that during the focus groups we were focusing on robotic pets for "keeping someone company."

Focus groups began with a drawing prompt where participants were asked to draw or write a few words about their ideal robotic pet. This was done to engage participants in a way that would get them thinking of the questions they had about robotic pets as well as obtain initial impressions before they interacted with other participants. This also gave participants a way to write about feelings or thoughts that might be perceived as stigmatizing if voiced out loud (e.g. feeling lonely and therefore desiring a robotic pet). Next, participants were asked questions such as what they thought of the idea of having a robotic pet, whether they would consider having one, whether they would want to interact with one, and how they would want to interact with it. Participants were also asked about any perceived concerns such as privacy, maintenance, and pets replacing human contact. Partway through each of the focus groups, we introduced six commercially available robotic pets to give participants concrete ideas of possibilities of robotic pets (see Figure 1). Each robot was passed around to participants, who were told the features of each robot (e.g., responds to touch). It is important to note that none of these robotic pets were designed for older adults, as the ones that are (e.g., Paro) are cost prohibitive. Additionally, the goal of introducing these robots was to stimulate conversation and provide participants with concrete examples that varied along dimensions of interaction type and appearance. Participants were told that these robotic pets were not designed for adults and to use them to brainstorm, provoke feedback, and discuss how seeing them affected their perceptions. During focus groups, we intentionally went back and forth between real pets and robotic pets (e.g. asking benefits of one over the other). All procedures were approved by the University's Institutional Review Board.

\section{Data Analysis}

Focus groups were audio recorded, transcribed, and verified by a member of the research team. As groups were not videorecorded, demographic information for individual quotes is not provided. Data analysis was conducted through a grounded theory approach [13]. Two members of the research team open-coded the transcripts independently to generate codes and grouped them into higher level themes. These codes were then selected and merged to create a codebook. The rest of the transcripts were then coded with 


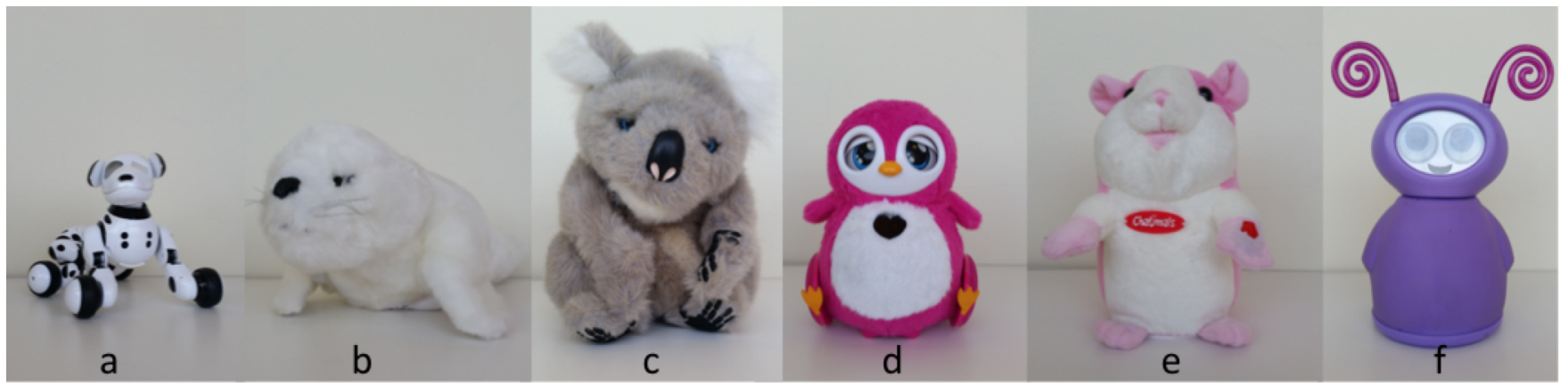

Figure 1. Robotic Pet examples shown during focus group: (a) Zoomer interactive puppy, (b) WowWee Alive Seal Pup, (c) WowWee Alive Koala Joey, (d) iloveRobots Penbo, (e) Chatimals, (f) Fijit Friends Willa

this codebook, which was updated to reflect emerging themes. The research team then related the codes to each other through an iterative process of memoing and theorizing, engaging in constant comparison of data to understand and refine a set of high-level themes.

This approach requires reflecting on our position as researchers and the perspective we bring to the analysis. We keep in mind previous research that challenges the ways that older adults are currently perceived in robotics [20, 34, 41] and are receptive to alternative viewpoints and reactions to these stereotypes that are voiced by older adults.

\section{FINDINGS}

Our analysis focuses on older adults' attitudes and perceptions of robotic pets. Throughout the focus groups we encouraged older adults to compare and contrast the qualities of real pets as a way of drawing out opportunities for designing appealing robotic pets. We identified three key tensions in the design of and current narrative around robotic pets: (1) deriving comfort and companionship from a robotic pet requires giving into the fiction of it, which is an older adult's choice; (2) rather than alleviating loneliness and social isolation, robotic pets may provide social entertainment and facilitate opportunities for social interaction; and (3) the functional support of robotic pets is appealing, but older adults value reciprocity inherent in caring for a pet and the relationship that it creates.

\section{Comfort and Companionship: Giving in to the Fiction}

Reflecting on their experience with real pets, participants articulated the connection and comfort they felt when physically interacting with a pet like a dog or cat. They noted that petting, snuggling with, and cuddling pets was a comforting experience and beneficial for mental health. A participant mentioned that interacting physically with pets satisfies a basic human need to connect to others:

"[Another participant] was talking about the furriness and the softness. Well there is this live creature there that will allow you to reach out in that way. Sometimes we don't do that to each other, [we] have a hard time, barriers and stuff. But animals are wide open to that kind of reception... [it's] a need that people have... to be connected in that way and have that tactile response."

Some participants at first assumed that robotic pets could not meet these needs due to conceptions of robots as hard and metallic. After the soft robotic pets were passed around, some participants changed their minds.

"Looking at these things, I see you have warm and fuzzy ones. I was thinking only of metallic which does not appeal to me at all. So something I could hug and cuddle maybe would be much more acceptable to me than a metal thing."

Other participants noted the unique nature of soft fur and how it was conducive to petting: a participant spoke about how she wanted a robotic pet "with fur... There's something about petting. I would want something with fur. I wouldn't want plastic." In addition to soft fur, the shape of a robotic pet affected participants' perceptions of its cuddliness. When the seal was passed around during a focus group, one participant noted that "Everyone is falling in love with it." When we asked why this was the case, another participant responded that "the way that the flippers are, they just kinda hug you." Indeed, we saw many participants holding and hugging the seal and the koala, which are the softest of the pets that we brought to the focus groups. One participant said, "Would you like to be held? Would you like to be in my arms?" Another participant said to the seal, "Could we be friends? Good, good. I love your eyes." This participant and others talked to the robotic pets and interacted with them in a playful and affectionate way.

Participants also spoke of sleeping with live pets and wanting a robotic pet to sleep with them as well (see Figure 2). One participant explained, "Well I would want one that would jump up on my lap like a cat does. And sleep with me like a cat does." Other participants mentioned being greeted at the door by a pet and its ability to transform a mood.

"My dog is always a happy hello. Right when you get that brightness, they're welcoming. Even if you are upset or angry or sad or whatever and it has positive impact." 


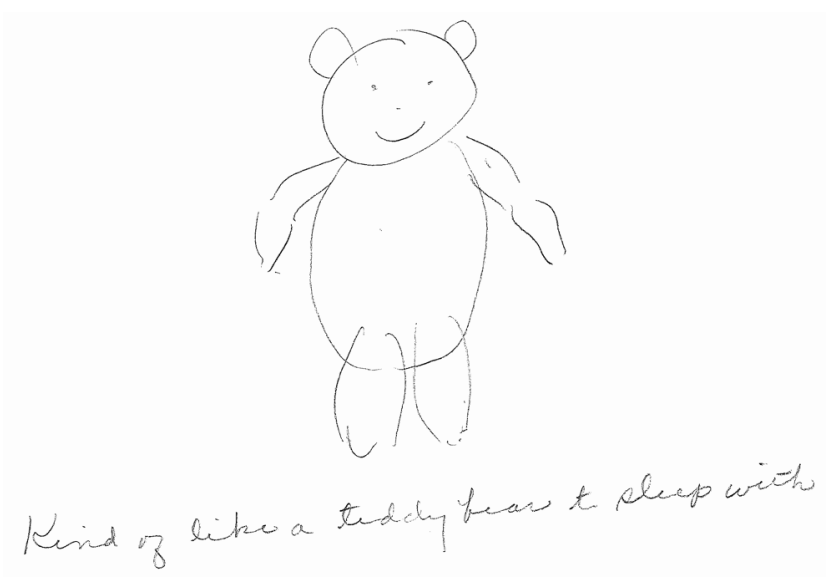

Figure 2. Ideal companion pet drawn by participant with description "kind of like a teddy bear to sleep with."

Some participants wanted robotic pets to provide a similar reaction to them, as in [43]. One participant wrote on her drawing page that her ideal robotic companion would "greet me when I return home with wagging tail and happy bounce." The participants did not view these robotic pets as replacing their social contacts or solving any kind of loneliness, but rather providing a warm and welcoming reaction when they returned home from other activities in their life. In addition to an initial greeting, many participants desired the robotic pet to listen and respond to them.

"Just to be a warm reaction to something... Like he's hearing what I'm saying."

"Well, you know, I don't want to do philosophical conversations with it, but just a little bit. Some reactions when you say, oh boy it's raining outside, and it could go, oh yeah. Just a little bit of-" Another participant interjects: "Small talk."

Though some participants wanted their pet to respond to them verbally, others preferred that the pets would not engage in conversation and only make noises and responses like live pets do. One participant explained wanting to hear noises rather than conversations, "Just so you know it's alive and you're not alone." In addition to wanting robotic pets to listen to and respond to verbal interactions, participants wanted them to respond to their emotions. They spoke of live pets' remarkable ability to understand when they were feeling sad and respond appropriately:

"My dog, he can tell usually if I'm in a bad mood or sad. He'll come to me and he will just lay next to me... I will just rub his head, and he will just stay here. He can tell when I'm in bad mood. He can tell when I'm angry too."

Although many participants were positive about the idea of a robotic pet as a companion who cuddles or greets an owner upon returning home, some were skeptical as to whether they could ultimately be a companion. If serving as a companion, the idea of whether a robotic pet should (or even could) respond to an older adult's mood was a source of tension for some participants, who doubted that a robot could ever react authentically like an actual pet.

"Feelings and emotion, you cannot give to a robot. You cannot put the feelings and emotion same like a real dog. You cannot put these things in a robot dog, I think."

Participants discussed that the robot did not act authentically out of its own needs and desires, but rather would be programmed to act as if it could respond to emotions.

"You could program one to act like it cares if you're crying. But it's just bullshit and you know it was bullshit. They're empty. They're nothing... It's just beyond my comprehension that anyone would ever want one."

"Just when you say the word companion, to me that implies the ability to notice that you're there and you care about them. Yes, robot could be programed and noticed that you're there or not. But, so what?"

"They say a dog is man's best friend. A computer isn't. It's your best friend because you've got it computerized to be your best friend. A dog will know you... you're its buddy and that's from it's heart. Computer's [friendship is] from its computer chip."

Hence, we observed a juxtaposition of the actions of some focus group participants, which suggested that they are open to petting, cuddling, sleeping with, and potentially deriving comfort from a robotic pet, and the feelings of others who vehemently described the artificial nature of the robotic pet as limiting its ability to be a true companion. The idea of developing a relationship with a robotic pet was viewed as "creepy" when understood in the context of real pets.

"It could be creepy, the notion... that I could expect it could react to me... That I might develop some affection for it."

"I just get a sick feeling in my stomach when you said create relationships with these things. That sounds like psychosis. It's a thing. It's no more human than that box of cookies"

"I would say [seeing examples of the robotic pets] confirms that unimaginable thing of being attached to it, or having love and affection for whatever this furry or plastic thing is. No matter what they're saying or doing, they're things. It confirms things are things."

The source of this tension is that participants conceived of the robotic pets as "things" incapable of being as real as an actual pet. As part of this, some participants objected to using the term "pet", as the concept of a pet implies life and the ability to form a relationship with its owner:

"I think the problem for me is just calling them pets... the word pet implies a lot of things. Most basically it implies life. That thing is just a glorified computer is all it is. I don't confuse my cat with my apple or computer. One doesn't replace the other. It's fun to have both of them. They don't serve the same purpose even remotely" 
"I guess I don't exactly think of it as a pet. I think of it as something I'd like. Definitely I'd like.”

To further explore this, we temporarily replaced the word "pet" with something else - one group of participants made up the word "Figrit" to use instead - and then continued discussion of whether it is possible to have a relationship with a robot. After acknowledging the artificial nature of robotic pets, some participants said that they believed they could still form relationships with one. However, they made it clear that this relationship was not similar to what one would have with a live being, but rather, akin to relationships with stuffed animals, cars, and movie characters.

"I had two stuffed animals, that I treated like they were alive... Can't have an animal anymore but I have a rapport with my stuffed animals."

“... a stuffed animal, you know that's not a real animal, but they can be quite nice to have around."

“... what's real is really up to me. That's how I see things. Often, some of the staff on some of the Star Trek movies feel almost like a part of my family. Obviously, they're characters made up by Gene Roddenberry and being played by actors. But I get fulfillment and interest and actually emotional connection even thought it might be considered one way."

Participants described having relationships with other inanimate objects, through which they have rapport, derive fulfillment, and achieve an emotional connection - albeit one-way. They expected a relationship with a robotic pet to be similar and reflected on their active role in defining that relationship as an imagined thing and a helper.

"Well, it would be a toy, but maybe an imagined thing.... Probably more like a toy but having a relationship that was quite fun with it. This is my helper. It's a robot but it's like a dog. And not be concerned about it. It would be a stylized relationship, but it could be a relationship."

What is critical here is that participants made it clear that they were the ones in charge of deciding what was real, what was artificial, and whether they would allow themselves to form a relationship with it. They described this as forgetting that it is a robot, living with an illusion, and accepting the fiction.

“After a while you probably forget that wasn't robotic with programmed in, maybe. And then you could buy into that... you could probably get to the point where you have a relationship with this non-biological, I don't know what this thing is made of, but you know-maybe you could get into, it's, sure, living with an illusion- we live with them anyway..."

"A companion responds to you emotionally. How can a robot be emotionally responding that you could believe it, unless you accepted the fiction."

Just as these older adults describe forming a relationship with other inanimate objects, some were willing to "accept the fiction" of a robotic pet and form a different kind of relationship with it than they would with a live pet. This echoes Alač's notion of our ability to view robots' social capabilities not in terms of our understanding of social rules from other humans, but rather, with them being a 'thing' [1]. As these participants explained, relationships with inanimate objects can still involve rapport and bring fulfillment and an emotional connection - as long a you are willing to accept the fiction of it.

\section{Social Opportunities rather than Social Isolation}

Stemming from the idea of having a relationship with a robotic pet as a companion, much of the current robotics literature [20] and popular press (e.g. [6]) conceptualizes robotic pets as a way of keeping older adults company and preventing loneliness. A few participants in our focus groups did speak of how a robotic pet could address issues of loneliness and social isolation. One participant wrote on the drawing probe that she wanted a robotic pet to "be there to keep me company. I feel lonely in my apartment. It would be lovely to have a 'substitute' pet." Another explained, "I would want it to be like a buddy. Where I wouldn't feel like a complete fool talking to it, " suggesting an awareness of how others perceive his interacting with it as a companion and his own perceived stigma associated with robotic pets.

However, many more participants did not see a robotic pet as remedying their loneliness because they did not feel lonely or isolated.

"I can't imagine having one in my present condition now. I'm very social. I have a lot to do... I guess if I was really lonely and didn't have a lot of friends and needed something then maybe one of these could fit into my living pattern."

"I have a husband so I don't have a big need for a companion animal right now."

Instead, many participants suggested that robotic pets would be good for alleviating loneliness among people who are "much older" and "elderly", similar to [34]. Participants brought up health conditions in which a robotic pet might help alleviate loneliness, such as mobility impairments that restrict a person's ability to go outside and being ill in the hospital with conditions such as cancer.

When positioned as a solution for reducing social isolation, the vast majority of participants were not interested in the devices. In contrast, participants shared many ideas regarding the potential of robotic pets as social entertainment and helping stimulate new opportunities for social interaction. These participants did not relate to the concept of a robotic pet as an all-encompassing solution to remedy isolation, but rather as an element to weave into the areas of their lives where social interaction was desired.

Participants spoke about the qualities of real pets that lead to entertainment. They valued traits such as a pet being "mischievous," "adventurous," "humorous" and even spoke fondly of a pet giving "aggravation" and being "erratic." A 
participant explained that a robotic pet needed to be erratic, as "it would be boring otherwise."

Participants wanted to interact playfully with pets by playing Frisbee, hide-and-seek, and having them follow commands. Even for a "lonely" person, participants thought robotic pets would provide the individual with activities through playful interaction.

"If it actually interacted, I think that's a big advantage for a lonely person. You could make it walk around the room or jump or something that would give you something to do",

In one group, a participant shared his vision of an ideal robot:

"I think with technology now they can have just about anything. We've all probably seen some sci-fi movies where you have the artificial intelligence, so it's appealing to that sense. I don't know if anybody saw that movie [Robot \& Frank].. it's this older guy, senile, that lives in upper state New York... He's an ex jewel thief, this guy, and he teaches this [robot] to pull a job with him... It had the capacity to think and remember and do what he said. That would be fun. That would be a blast."

This participant imagined enjoying an interaction with a robot abetting him in carrying out robberies. Though this interaction itself would be challenging to design (and illegal), the mischievous and fun personality of the robot and the resulting experience is an interesting avenue for design.

Some found the more talkative and responsive robots engaging. One participant said to the chipmunk, "You're very cute and you're very entertaining." However, others grew tired of the entertainment aspect of the example robotic pets, suggesting the novelty would wear off quickly.

"Well it does the same thing... and it probably is gonna do the same stuff over and over and then you get tired of it, the novelty of it."

"It gives you laugh, but it's the same laugh because it does the same thing, its not spontaneous. You get tired of it quick."

In addition to their own entertainment, participants viewed having a robotic pet as a way of enhancing interactions with other people. For example, one participant wrote on the drawing probe that she'd like to have a robotic pet as "a topic of conversation [with] my husband who also likes dogs." Participants drew on their experience with real pets and suggested that a robotic pet could be a way of meeting new people and giving you things to talk about with them.

"I have a dog who is kind of unique, she's very small and she's very friendly and it really promotes [interactions with others]. People would stop their cars to ask me what kind of dog it is. And I imagine, if you want to have more interaction with people you should have a pet that other people want to talk about."

"Well, like our dog park, it's a good way for people to get together and talk and everything. Because they have something in common at least, at first, the dogs. And so it's a good way to meet the neighbors or maybe find a friend."

Some participants spoke of taking robotic dogs to the park where they could play with the live dogs and one participant even mentioned using the robotic dog to meet people to date. Commenting on the potential for social interaction, one participant said, "I think you would have wonderful parties."

In summary, the vast majority of participants eschewed the idea of a robotic pet as something that could alleviate their own social isolation [34]. Instead, these independent older adults envisioned varied ways in which robotic pets could provide social entertainment and help them stay socially engaged by enabling them to meet others.

\section{Balancing Functional Benefits with Reciprocity}

Throughout the focus groups, participants described many functional benefits of robotic pets that provide advantages over real pets. One key advantage cited for robotic pets is that they do not need the level of care that a live pet requires [46]. Participants acknowledged that owning a pet later in life is more difficult because of the care it requires and noted that a robotic pet is appealing for this reason.

"But I think when I'm quite a bit older, even though I knew it wasn't a real dog, when I wasn't able to do the things that [other participant] said to take care of a real dog, then I think it could be quite nice."

Robotics are also touted as being useful for older adults because of the potential to provide functional assistance while aging in place [39]. Participants suggested that robotic pets could provide them with assistance in various ways based on their understanding of both real pets and robotic capabilities. For example, participants suggested that a robotic dog could alert its owner to a knock on the door when the owner had hearing impairments or serve as security and an alarm in case of break ins. Participants also came up with highly imaginative and novel uses for robotic pets, such as carrying home groceries, finding slippers, doing the dishes, and retrieving objects from hard to reach places (Figure 3).

Some participants who were unable to imagine developing a relationship based on social interactions with a robotic pet were interested in the idea of a robotic pet for functional assistance, such as a participant who said "Attachment [can happen] with a real dog, and a robot, we don't have that. But they can work for us." Another participant responded to others listing some of the functional assistance they'd like the robot to provide, saying "So basically we need one that is a maid." This interest in a robot to serve as a maid or butler for the general population has been explored in [16].

Other participants, however, saw functional assistance as "woven in" with the relationship formed with a pet, robotic or live. That is, participants saw reciprocity as a virtue of pets; the give-and-take nature of caring for a pet and having the pet provide assistance or comfort in return is important. While participants rejoiced at the notion of not having to put 


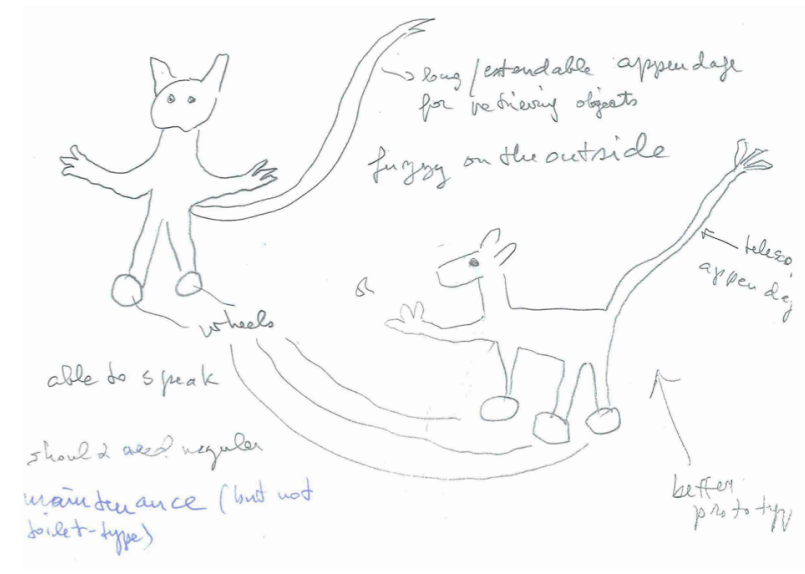

Figure 3. One participant envisioned a robotic pet with a "long/extendable appendage for retrieving objects" utilizing the pets tail as the appendage.

up with incessant yapping, and, particularly, not clearing out litter boxes or cleaning pet messes, they also longed for the attachment that comes from caring for a pet. Surprisingly, participants spoke of wanting to do things for the robotic pets. They valued the reciprocal nature of their relationship with a pet, which is achieved by caring for the animal and deriving some benefit in return. Importantly, participants described the work one does for a pet as a way of bonding with it and suggested that they may use robotic pets longer if they require some level of maintenance. Further, one participant described how the robotic pet might get relegated to a corner of her house if it did not need maintenance:

"Would it just become one of my many stuffed animals I had since I was a child or would it just be another piece of clutter in my house? I'm not quite sure where the bonding would come because you don't feel like you have to feed it, nourish it, or cuddle it..."

In contrast to work which asserts that older adults may be overwhelmed by caring for a robotic pet [25], participants said that the robotic pet should have a need for maintenance built into it. One participant suggested having to interact with a robotic dog every day for it to work and said this idea appealed to "the nourishing part of me." And, participants noted that providing care for an animal leads to attachment.

"I think being responsible for something makes you more attached to it... [because of the] feeling that it needs you in order to function. And pets do need you."

Another reason that participants wanted to care for the robotic pet was to encourage them to be active and engaged with the world. The reciprocity inherent in pet ownership helps some owners feel needed and a sense of purpose.

"My daughter used to say, everyone needs to be needed. And if a pet, even robotic, needs to be cared for in some way, then that gives me a reason to get up in the morning and give the pet a breakfast.",
Other participants spoke of how a live dog must be walked outside of the house, thereby forcing the owner to exercise. They spoke of wanting a robotic pet that needed to be walked so that they would have to get exercise. Even though one of the main reasons robotic pets are said to be appealing to older adults is the lack of maintenance, we found that participants were interested in maintaining the pet in certain ways, both in order to build the relationship as well as encouraging them to stay active. For many of the participants, a one-way relationship where the pet provided all the support and did not require anything in return was not desired.

\section{DISCUSSION}

Robotics research and design has primarily focused on a stereotypical view of the older adult as lonely and in need of extensive functional assistance [34]. Indeed, when we asked about robotic pets as a tool to alleviate social isolation, participants saw them as useful for other people who were "older" and "elderly." The literature suggests that when older adults view a device or product as for people who are older or more frail then they are, they are perceiving the stigma associated with these devices and stereotypical views of aging as decline and isolation [21, 34]. Stereotypical assumptions about older adults affect the design of the robots that have been created [41], but do not accurately represent the diversity of interests and preferences among older adults. Further, older adults are often "considered but not consulted" in the design of robots [20]. In this study, we use focus groups to provide older adults with a platform to discuss their views on robotic pets, which contributes to the ongoing discussion of the ethics of robotic companions for older adults and provides new insights into older adults' preferences for interaction with robotic pets.

\section{Ethics of Robotic Companions for Older Adults}

The ethics of introducing robotic companions to older adults is a widely debated issue across the fields of robotics, humancomputer interaction, and gerontology. The literature highlights two key ethical issues concerning robotic companions for older adults.

First, the debate over introducing robotic pets into the lives of older adults as a source of companionship is fueled by the assumption the older adults are (or will be) unable to understand the artificial nature of the robot and are encouraged to have a relationship with the robotic pet as if it were real. Inherent in this is the idea that these older adults are "tricked" into forming a relationship with something that is not real and "does not understand the arc of human life" [51]. Dautenhan asks, "Is it ethically justifiable to aim to create robots that people bond with, e.g. in the case of elderly people or people with special needs?" [15]. Sparrow specifies that the ethical issue is due to the benefit of a companion robot depending on the older adult believing that the robot [47] and, specifically, the robotic pet [46], can actually empathize with them and understand them.

Participants in our study were keenly aware of the tension between deriving comfort from a robotic pet and giving in to 
the fiction of it, but they also described acknowledging and accepting its artificial nature. That is, deriving benefits from a companion robot may mean intentionally accepting an illusion or alternate reality. Further, these older adults said that believing the fiction of the robot was a conscious choice that they could choose to make or not make: some wanted a robotic pet to sleep in bed with them, others called developing a relationship with a robot psychosis. But when distanced from the idea of an actual pet as a companion, participants opened up about other relationships in their life with inanimate objects, such as cars, stuffed animals, and TV characters, which they allowed themselves to embrace and benefit from. Importantly, participants saw themselves as actively shaping and stylizing their relationship with inanimate objects in a purposeful way. This perspective counters the stereotype of older adults being lured into bonding with a robot without understanding that it is not actually alive. Instead, older adults see themselves as capable and in control of making the decision to believe and thus derive benefits from a companion-oriented robotic pet.

A second ethical issue raised by researchers is that robots will replace human caregivers, thereby reducing the amount of contact that older adults will have with others [45, 47]. This assertion aligns with normative views of aging in which growing older is characterized by a period of declining health, increasing disability, and loneliness in which a caregiver may be an older adult's main (or only) source of social interaction. While this may be true for a subset of older adults, those in our study did not share this vision of what it means to grow older. Likely in part due to the stigma around robotic pets and social isolation, many participants spoke about how they had so many interactions with others that they did not need or have time for a robotic companion. Instead, participants perceived a robotic pet as a resource for fostering social entertainment and engaging socially with others (e.g., at a dog park or party). Participants rejected the idea of a robot as a replacement for humans and spoke about how they might integrate the robot into areas of their lives in which they had a momentary need for social interaction, such as a greeting when they walked in the door. Our data suggests that the current narrative of robotic companions replacing human social interaction misses an opportunity to design experiences with robotic companions that facilitate new social encounters, and we elaborate this point below.

Although we raise issue with some of the ethical arguments regarding the general population of older adults bonding with a robotic companion $[15,47]$, it becomes more complex when we consider individuals with cognitive impairments, who can be perceived as having challenges discerning between reality and fiction, or older adults who are isolated with most interaction coming from caregivers. The participants in this study lived independently and do not fit into the categories that many robots for older adults appear to have been designed for. When debating the ethics of robots, however, as a field, we must be mindful to not disempower older adults by treating older people as homogenous and implying that they, as a group, are lonely and dependent on caregivers for interaction, or that they are incapable of discerning on their own whether they desire to embrace the fiction and bond with a robotic companion.

\section{Design Considerations}

In addition to furthering the ethical discussion around robotic pets in the lives of older adults, our analysis provides guidance for future design work on companion pet-like robots. Here we revisit our three high-level findings and discuss the design considerations of each.

\section{Comfort and Companionship: Giving in to the Fiction}

Participants spoke about the beneficial aspect of cuddling and petting live pets and many expressed interest in a robotic pet that was warm, soft, and in a shape suitable for hugging and cuddling. Thus, one obvious recommendation is to pay attention to the forms that are tangibly appealing to adults. In addition to enjoying the tangible elements of cuddling, participants enjoyed having a pet respond to their actions and emotions. However, they questioned the capacity of a robotic companion to do this without making them feel "like a complete fool." Therefore, the researcher must figure out how to help participants give in to the fiction if they wish to do so. There are some basic recommendations, such as eliminating or quieting the sounds of the motors in the robotic pets, which many participants found to break the illusion. Other questions lead to future research questions: What kinds of interactions allow participants to buy into the illusion and which break this illusion? Do responses of other individuals and societal expectations, as well as the context of use affect whether an individual feels "like a fool" interacting with a robot? How should robotic pets respond so that they do not deviate too far from a conception of how an interactional partner might act, without falling into the trap of attempting to accurately imitate a live pet (i.e., the uncanny valley [32])? Young children can hold conceptions of a robot as an agent and a thing [1]; our analysis suggests that older adults may experience a similar dualism.

\section{Social Opportunities rather than Social Isolation}

Though much of the research on robotic pets has framed them as a solution for lonely older adults [20], only a few participants spoke of wanting a robotic pet because of loneliness. A more widely held desire was for a robot to entertain them and play with them. Though play has been a goal of robotics for other groups (e.g. families [18] and children with autism [48]), it has not been an emphasis for older adults, despite some evidence that enjoyment and fun do appear to affect older adults' acceptance and intention to use robots [3, 22, 55]. However, this prior research disregards the idea of a robot's main purpose as being for entertainment [22]. In contrast to the general population, older adults' playful and creative interactions with technology are seen by designers as errors [35]. Peine et al. explains that this is due to technology being designed in a paternalistic manner where the goal is for older adults to be "comforted rather than challenged" by technologies [35]. However, participants in focus groups spoke of wanting 
challenging interactions from mischievous, playful, idiosyncratic, and even aggravating robotic pets. Rethinking the design of robotic pets for older adults may mean forgoing designs that focus on being friendly and pleasing in favor of interactions that are more unpredictable and playful. Again, this opens up future research questions based on the expressed desires of focus group participants: how do you design a mischievous robot? What entertaining elements are seen as child-like and which are seen as appropriate for adults? What is an appropriate level of aggravation that a pet should produce? Additionally, the novelty effect has not yet been sufficiently studied with older adults [26]: What entertaining elements of a robotic companion remain entertaining and do not lose their novelty?

In addition to seeing the potential of a robot for entertaining themselves, participants came up with novel uses that have not been fully explored. Participants expressed interest in a robotic pet that, like a live pet does, would help them initiate social interactions and have a topic to talk about with strangers as well as friends and family. Though a robotic pet as a social facilitator has been explored in a nursing home in one study [25] and hinted at in findings when researchers are surprised at robots initiating interactions or a sense of community $[45,52,54]$, this concept has not been explored in the design of a robot for independently living older adults. Sacks [44], cited in [50], described how dogs serve as "tickets to talk," meaning people can start a conversation when walking a dog, when this might normally be seen as an unwelcome advance. We propose exploring what features of robotic pets can serve as "tickets to talk." In designing robots as social facilitators, some possible venues are having robots recognize other robots or pets and initiate play with them.

\section{Balancing Functional Benefits with Reciprocity}

There has been discussion in the literature about the importance of robot 'owners' contributing to the maintenance of a robot as a way of building a relationship [47]. And indeed, many commercially available robots have been toys that "play on people's need to express nurturance by requiring caretaking in order to thrive," such as the Furby and Aibo (robotic dog) [5]. However, for older adults, the lack of maintenance is stressed as a reason these robots are suitable for older adults [46], which is at odds with having people take care of them in a way that will help build a relationship [47]. Additionally, researchers have even warned of the emotional burden of having to care for a robotic pet: Dautenhahn claims that the caretaker paradigm of robot interaction, where a person's job is to 'identify and respond to the robot's emotional and social 'needs', would be too burdensome for an older adult to maintain and is therefore unethical [15]. Kidd et al. echo this assumption: although older adults enjoyed playing with Paro, the researchers inferred that older adults did not wish to own their own robotic seal because they did not want to take on the maintenance of tending to a robotic pet [25]. In contrast, our participants desired certain types of maintenance so that they could express nurturing and develop a reciprocal 'give and take' relationship. They also saw maintenance as being beneficial to themselves, such as contributing a sense of being needed and being forced to exercise. We question whether robotic pets should be "easy" to care for, thus depriving older adults of the work inherent in caring for a real pet and the sense of attachment that provides.

We recommend building the need for maintenance into interactions with robotic pets, a concept that has been discussed in [18]. One way to do so would be to follow one participant's recommendation to have a robotic companion require the older adult to interact with it everyday for it to function properly. Other ideas include designing a pet that will become irritating, grumpy or sad, or perhaps barking or even making (water soluble) 'messes' on the carpet if it is not taken out for a walk. Not tending to a real pet has real consequences, and participants did not want to be protected from some of the aggravating but integral elements of pets.

\section{CONCLUSION}

In the literature, most robotic pets designed for older adults either provide functional assistance or provide companionship to an older adult who is perceived as lonely and physically frail. Additionally, researchers have discussed perceived ethical issues such as robots tricking older adults to believe that they are real and the idea that robots will replace human interactions. By involving older adults in discussion through focus groups, we found that older adults were very aware of the ethical issues that come with robot use. However, they made it clear that they were active in deciding to (or not to) 'accept the fiction' in order to form a relationship with a robotic companion. Additionally, they rejected the idea of a robotic pet as a solution to loneliness, but welcomed the idea of having a robot to fill certain social needs that were not currently satisfied, or enhancing social interactions with others. Finally, participants did not want a robot that solely contributed functional assistance and did not require anything in return. They wanted to care for robotic pets as a way of expressing nurturance and building a relationship. Based on these insights, we provide design recommendations and future research questions to work towards rethinking robotic pets that are not seen as "technological fixes" for older adults, but are desired by them to suit certain needs and fit into existing lives that are already filled with rich social interactions.

\section{ACKNOWLEDGEMENTS}

This work was supported in part by the NSF Graduate Research Fellowship grant (DGE-1256082).

We thank the individuals who participated in this study and the following undergraduate researchers for their contributions: Maureen Fallen, Josephine Le, Jinjing Liu, and Olga Vitruk. 


\section{REFERENCES}

1. Morana Alač. 2015. Social robots: Things or agents? AI \& Society. Published online: 07 November 2015.

2. Marian R. Banks, Lisa M. Willoughby, William A. Banks. 2008. Animal-Assisted Therapy and Loneliness in Nursing Homes: Use of Robotic versus Living Dogs. Journal of the American Medical Directors Association 9, 3: 173-177.

3. Willy Barnett, Kathy Keeling, and Thorsten Gruber. 2015. Investigating User Perceptions of HRI: A Marketing Approach. In Proceedings of the ACM/IEEE International Conference on Human-Robot Interaction Extended Abstracts (HRI'15 Extended Abstracts), 1516.

4. Jenay M. Beer, Cory-Ann Smarr, Tiffany L. Chen, Akansha Prakash, Tracy L. Mitzner, Charles C. Kemp, Wendy A. Rogers. 2012. The Domesticated Robot: Design Guidelines for Assisting Older Adults to Age in Place. In Proceedings of the ACM/IEEE international conference on Human-Robot Interaction (HRI '12), 335-342.

5. Timothy W. Bickmore and Rosalind W. Picard. 2005. Establishing and maintaining long-term humancomputer relationships. ACM Transactions on Computer-Human Interaction 12, 2 (June 2005), 293327.

6. Ann Brenoff. Toy Cat Robots May Be Just The Thing For Lonely Seniors. Huffington Post Article. (19 December 2015). Retrieved January 13, 2016 from http:/www.huffingtonpost.com/entry/toy-cat-robotsmight-be-the-answer-to-seniorloneliness 564e0ee1e4b08c74b734c9b9.

7. Cynthia Breazeal. 2003. Toward sociable robots. Robotics and Autonomous Systems 42: 167-175.

8. Elizabeth Broadbent, Rie Tamagawa, Ngaire Kerse, Brett Knock, Anna Patience, Bruce Macdonald. 2009. Retirement home staff and residents' preferences for healthcare robots. In The 18th IEEE International Symposium on Robot and Human Interactive Communication. 645-650.

9. Joost Broekens, Marcel Heerink, Henk Rosendal. 2009. Assistive social robots in elderly care: a review. Gerontechnology 8, 2: 94-103.

10. Praminda Caleb-Solly, Sanja Dogramadzi, David Ellender, Tina Fear, Herjan van den Heuvel. 2014. A Mixed-Method Approach to Evoke Creative and Holistic Thinking about Robots in a Home Environment. In Proceedings of the ACM/IEEE international conference on Human-Robot Interaction (HRI '14), 374-381.

11. Wan-Ling Chang and Selma Šabanović. 2015. Studying Socially Assistive Robots in Their Organizational Context: Studies with PARO in a
Nursing Home. In Proceedings of the ACM/IEEE International Conference on Human-Robot Interaction Extended Abstracts (HRI'15 Extended Abstracts), 227228.

12. Wan-Ling Chang, Selma Šabanovic, and Lesa Huber. 2013. Use of seal-like robot PARO in sensory group therapy for older adults with dementia. In Proceedings of the ACM/IEEE international conference on Humanrobot interaction (HRI '13), 101-102.

13. Kathy Charmaz. 2006. Constructing grounded theory: a practical guide through qualitative analysis. SAGE Publications.

14. Diego Compagna and Florian Kohlbacher. 2015. The Limits of participatory technology development: The case of service robots in care facilities for older people. Technological Forecasting and Social Change 93, 1931.

15. Kerstin Dautenhahn. 2007. Socially intelligent robots: dimensions of human-robot interaction. Phil Trans $R$ Soc B 362: 679-704.

16. Kerstin Dautenhahn, Sarah Woods, Christina Kaouri, Michael L. Walters, Kheng Lee Koay, Iain Werry. 2005. What is a Robot Companion - Friend, Assistant, or Butler? In IEEE/RSJ International Conference on Intelligent Robots and Systems, 1192-1197.

17. Jeannette Durick, Toni Robertson, Margot Brereton, Frank Vetere, Bjorn Nansen. 2013. Dispelling Ageing Myths in Technology Design. In Proceedings of the 25th Australian Computer-Human Interaction Conference: Augmentation, Application, Innovation, Collaboration (OzCHI '13), 467-476.

18. Ylva Fernaeus, Maria Håkansson, Mattias Jacobsson, and Sara Ljungblad. 2010. How do you play with a robotic toy animal?: a long-term study of Pleo. In Proceedings of the 9th International Conference on Interaction Design and Children (IDC '10), 39-48.

19. Jodi Forlizzi, Carl DiSalvo, and Francine Gemperle. 2004. Assistive robotics and an ecology of elders living independently in their homes. Human-Computer Interaction 19, 1 (June 2004), 25-59.

20. Susanne Frennert and Britt Östlund. 2014. Review: Seven Matters of Concern of Social Robots and Older People. International Journal of Social Robotics 6, 2: 299-310.

21. Leonardo Giusti, Eleonora Mencarini, and Massimo Zancanaro. 2010. "Luckily, I don't need it": elderly and the use of artifacts for time management. In Proceedings of the 6th Nordic Conference on HumanComputer Interaction: Extending Boundaries (NordiCHI '10), 198-206.

22. Marcel Heerink, Ben Kröse, Bob Wielinga, and Vanessa Evers. 2008. Enjoyment intention to use and actual use of a conversational robot by elderly people. 
In Proceedings of the ACM/IEEE international conference on Human robot interaction (HRI '08), 113120.

23. Marcel Heerink, Jordi Albo-Canals, Meritchell ValentiSoler, Pablo Martinez-Martin, Jori Zondag, Carolien Smits, Stefanie Anisuzzaman. 2013. Lecture Notes in Computer Science, 104-115.

24. Suzanne Hutson, Soo Ling Lim, Peter J. Bentley, Nadia Bianchi-Berthouze, Anne Bowling. Investigating the Suitability of Social Robots for the Wellbeing of the Elderly. Affective Computing and Intelligent Interaction. Design for Aging. Volume 6974 of the series Lecture Notes in Computer Science, 578-587.

25. Cory D. Kidd, Will Taggart, Sherry Turkle. 2006. A Sociably Robot to Encourage Social Interaction among the Elderly. In Proceedings of the 2006 IEEE International Conference on Robotics and Automation, 3972-3976.

26. Reza Kachouie, Sima Sedighadeli, Rajiv Khosla, MeiTai Chu. 2014. Socially assistive robots in elderly care: a mixed method systematic literature review. International Journal of Human-Computer Interaction.

27. Rajiv Khosla, Mei-Tai Chu, Reza Kachouie. 2012. Embodying Care in Matilda - An Affective Communication Robot for the Elderly in Australia. In Proceedings of the 2nd ACM SIGHIT International Health Informatics Symposium (IHI '12), 295-304.

28. Stephen C. Kramer, Erika Friedmann, Penny L. Bernstein. 2009. Comparison of the Effect of Human Interaction, Animal-Assisted Therapy, and AIBOAssisted Therapy on Long-Term Care Residents with Dementia. Anthrozoös 22, 1: 43-57.

29. Alexander Libin and Jiska Cohen-Mansfield. 2004. Therapeutic robocat for nursing home residents with dementia: Preliminary inquiry. American Journal of Alzheimer's Disease and Other Dementias 19, 2: 111116.

30. Sean A. McGlynn, Shawn C. Kemple, Tracy L. Mitzner, Chih-Hung King, Wendy A. Ragers. 2014. Understanding Older Adults' Perceptions of Usefulness for the Paro Robot. In Proceedings of the Human Factors and Ergonomics Society 58th Annual Meeting, 1914-1918.

31. Ádám Miklósi and Márta Gácsi. 2012. On the utilization of social animals as a model for social robotics. Frontiers in Psychology 19, Article 75.

32. Masahiro Mori (Translated by Karl F. MacDorman and Norri Kageki). 2012. The Uncanny Valley. IEEE Robotics and Automation Magazine 19, 2: 98-100.

33. Toshiharu Mukai, Shinya Hirano, Hiromichi Nakashima, Yo Kato, Yuki Sakaida, Shijie Guo, Shigeyuki Hosoe. 2010. Development of a NursingCare Assistant Robot RIBA That Can Lift a Human in
Its Arms. In Proceedings of the IEEE/RSJ 2010 International Conference on Intelligent Robots and Systems (IROS 2010), 5996-5601.

34. Louis Neven. 2010. 'But obviously not for me': Robots, laboratories and the defiant identity of elder test users. Sociology of Health and Illness 32, 2: 335-347.

35. Alexander Peine, Ingo Rollwagen, Louis Neven. 2014. The rise of the "innosumer"- Rethinking older technology users. Technology Forecasting and Social Change 82, 199-214.

36. Martha E. Pollack, Laura Brown, Dirk Colbry, Cheryl Orosz, Bart Peintner, et al. 2002. Pearl: A Mobile Robotic Assistant for the Elderly. In Workshop on Automation as Caregiver: the Role of Intelligent Technology in Elder Care (AAAI), 85-91.

37. Akanksha Prakash, Jenay M. Beer, Travis Deyle, CoryAnn Smarr, Tiffany L. Chen, Tracy L. Mitzner, Charles C. Kemp, and Wendy A. Rogers. 2013. Older adults' medication management in the home: how can robots help? In Proceedings of the ACM/IEEE international conference on Human-robot interaction (HRI '13), USA, 283-290.

38. Akanksha Prakash, Charles C. Kemp, and Wendy A. Rogers. 2014. Older adults' reactions to a robot's appearance in the context of home use. In Proceedings of the ACM/IEEE international conference on Humanrobot interaction (HRI '14), 268-269.

39. Hayley Robinson, Bruce MacDonald, Elizabeth Broadbent. 2014. The Role of Healthcare Robots for Older People at Home: A Review. International Journal of Social Robotics 6, 4: 575-591.

40. Yvonne Rogers and Gary Marsden. 2013. Does he take sugar? Moving beyond the rhetoric of compassion. interactions 20, 4: 48-57.

41. Selma Šabanović. 2010. Robots in Society, Society in Robots. International Journal of Social Robotics 2, 4: 439-450.

42. Selma Šabanović, Wan-Ling Chang, Casey C. Bennett, Jennifer A. Piatt, David Hakken. 2015. A Robot of My Own: Participatory Design of Socially Assistive Robots for Independently Living Older Adults Diagnosed with Depression Human Aspects of IT for the Aged Population. Design for Aging. Volume 9193 of the series Lecture Notes in Computer Science, 104114.

43. Alessandra Maria Sabelli, Takayuki Kanda, Norihiro Hagita. A Conversational Robot in an Elderly Care Center: an Ethnographic Study. In Proceedings of the ACM/IEEE international conference on Human-Robot Interaction (HRI '11).

44. Harvey Sacks. 1992. Lectures on Conversation. Basil Blackwell, Oxford. 
45. Amanda Sharkey and Noel Sharkey. 2012. Granny and the robots: ethical issues in robot care for the elderly. Ethics and Information Technology 14, 1:27-40.

46. Robert Sparrow. 2002. The march of the robot dogs. Ethics and Information Technology 4, 4: 305-318.

47. Robert Sparrow and Linda Sparrow. 2006. In the hands of machines? The future of aged care. Minds and Machines 16, 2: 141-161.

48. Cady M. Stanton, Peter H. Kahn Jr., Rachel L. Severson, Jolina H. Ruckert, and Brian T. Gill. 2008. Robotic animals might aid in the social development of children with autism. In Proceedings of the $3 \mathrm{rd}$ ACM/IEEE international conference on Human robot interaction (HRI '08), 271-278.

49. Norman Makoto Su, Leslie S. Liu, and Amanda Lazar. 2014. Mundanely miraculous: the robot in healthcare. In Proceedings of the Nordic Conference on HumanComputer Interaction: Fun, Fast, Foundational (NordiCHI '14), 391-400.

50. Marcus Sanchez Svensson and Tomas Sokoler. 2008. Ticket-to-talk-television: designing for the circumstantial nature of everyday social interaction. In Proceedings of the Nordic conference on Humancomputer interaction: building bridges (NordiCHI '08), 334-343.
51. Sherry Turkle. Connected, but alone? Video. (February 2012). Retrieved January $12^{\text {th }}, 2016$ from

https://www.ted.com/talks/sherry_turkle_alone_togethe r?language $=$ en

52. Sherry Turkle, Will Taggart, Cory D. Kidd, Olivia Dasté. 2006. Relational artifacts with children and elders: the complexities of cybercompanionship. Connection Science 18, 4: 347- 361.

53. John Vines, Gary Pritchard, Peter Wright, Patrick Olivier, Katie Brittain. 2015. An Age-Old Problem: Examining the Discourses of Ageing in HCI and Strategies for Future Research. ACM Transactions on Computer-Human Interaction, 22, 1: Article 2.

54. Kazuyoshi Wada and Takanori Shibata. 2007. Living With Seal Robots-Its Sociopsychological and Physiological Influences on the Elderly at a Care House. IEEE Transactions on Robotics 23, 5: 12501255 .

55. James E. Young, Richard Hawkins, Ehud Sharlin, Takeo Igarashi. 2009. Toward Acceptable Domestic Robots: Applying Insights from Social Psychology. International Journal of Social Robotics 1, 1: 95-108. 